\title{
Solving combined geospatial tasks using 2D and 3D bar charts
}

\author{
Stefan Seipel ${ }^{* \dagger}$ and Leonor Carvalho ${ }^{\ddagger}$ \\ * Department of Industrial Development, IT and Land Management \\ University of Gävle, Sweden \\ Email: ssl@hig.se \\ $\dagger$ Centre of Image Analysis \\ Uppsala University, Sweden \\ $\ddagger$ University of Turku, Finland \\ Email:leofer@utu.fi
}

\begin{abstract}
This paper presents a user study that investigates 2D and 3D visualizations of bar charts in geographic maps. The task to be solved by the participants in this study required estimation of the ratio of two different spatial distance measures and relative ranking among potential candidates. The results of this experiment show that subjects were equally fast and accurate when using both the $2 \mathrm{D}$ and $3 \mathrm{D}$ visualizations. Visual discomfort was reported by almost half of the test population, but performance was not affected. Our study also showed that frequent game players did not benefit more from a 3D visualization than inexperienced game-players.
\end{abstract}

Keywords-3D visualization; geovisualization; evaluation;

\section{INTRODUCTION}

In many situations advanced 3D visualization and interaction techniques have been shown to support users in performing complex spatial tasks more efficiently. Also in geographical information systems (GIS) the use of 3D visualization appears as a natural way to present information in spatial context [1]. Advanced device technology needed to accomplish 3D visualizations, as e.g. stereoscopic displays and 3D head tracking, is meanwhile readily available at consumer level costs. In consequence, when building geographical information systems, the graphical design is likely to be influenced by intuition and technical finesse. Instead, it should be guided by an understanding of the kind of tasks that benefit from the possibilities offered by $3 \mathrm{D}$ visualization [2], [3].

The objective of the study presented in this paper is to compare the relative value of $2 \mathrm{D}$ and $3 \mathrm{D}$ visualizations of quantitative information in geographic context and to analyze potential effects based on other factors in the studied population.

\section{RELATED WORK}

$3 \mathrm{D}$ visualizations in geographical context are quite selfevident in situations where the content to be visualized has naturally $3 \mathrm{D}$ spatial properties. For instance, in urban planning [4] or in landscape planning [5] the content to be visualized are realistic structures which are to be presented in an authentic way. In this context geovisualization is not different from other applications of photo-realistic visualizations such as games or simulator based training.

In geospatial data-analysis on the other hand abstract information, often numbers, is presented and evaluated in its geographical context, whereby cartographic maps provide geographic reference frames [6]. Insight is thereby gained from seeing relations between quantities and patterns as they become evident in their geographic neighborhood. The well-known visualization of the Cholera epidemic in London in 1854 [7] is one illustrative example which incidentally demonstrates a very efficient $2 \mathrm{D}$ graphical design. The use of 3D maps for visualization of abstract i.e. non-spatial information has been popularized in geovisualizations already in the 1970 s with the introduction of the 3D space-time cube by Hägerstrand [8]. The concept of the space-time cube has recently been evaluated for spatio-temporal analysis in various applications [9], [10], [11].

\section{A. $2 D$ and $3 D$ visualizations}

In the visualization literature various notions exist for different types of 3D visualizations. According to computer graphics textbooks a projection of a model represented in $3 \mathrm{D}$ onto a $2 \mathrm{D}$ projection plane is to be considered a $3 \mathrm{D}$ rendering as long as it provides some of the so-called monoscopic depth-cues (linear perspective being one of them). According to this concept, even a planar (i.e. 2D) map defined as a flat planar surface in 3D can be considered a $3 \mathrm{D}$ visualization if it is projected back on the $2 \mathrm{D}$ computer screen at some angle and with some perspective distortion. This notion has been assumed by among others Lind et al. [12] who investigated traditional 2D user interfaces for industrial control that were virtually slanted in 3D. These 3D visualizations are based on monoscopic depth-cues only and they fail to exploit other highly efficient 3D cues such as motion parallax (e.g. due to dynamic observer position) as well as stereoscopic parallax [13]. Following the terminology in [14] we use in our study the following definitions for our visualizations: 


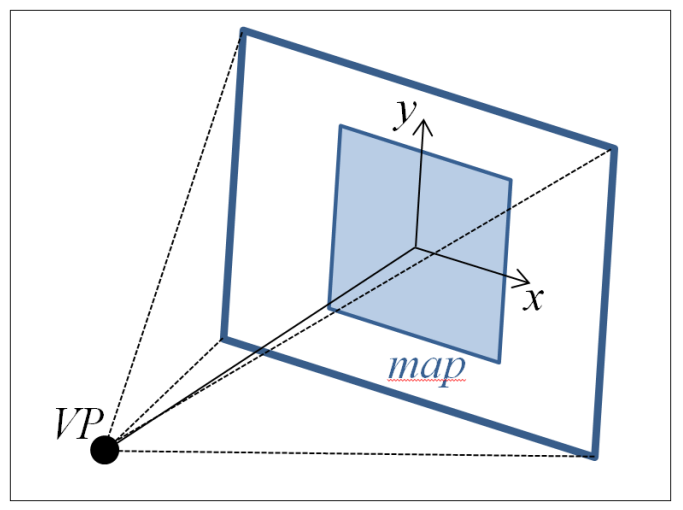

(a)

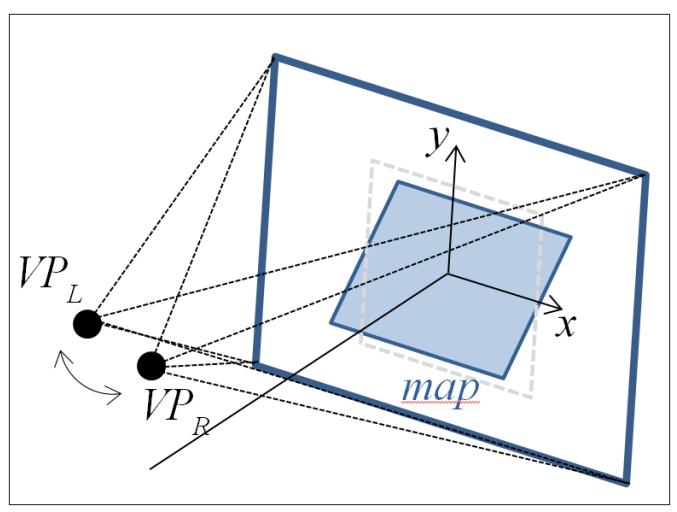

(b)

Figure 1. Illustration of the conceptual design of (a) a $2 D$ visualization and (b) a strong $3 D$ visualization of maps.

- A $2 D$ visualization is conceptually defined as a twodimensional geometry which is positioned in and aligned with the 2D plane of the display. Even for a stereoscopic rendering using some viewpoint dependent perspective projection, the $2 \mathrm{D}$ visualization will not create any depth cues (neither linear perspective nor parallax). See Figure 1(a).

- A strong $3 D$ visualization is conceptually defined by geometry in 3D space which is rendered using both monoscopic and stereoscopic depth cues. Dynamic 3D cues (motion parallax) are provided even for static objects since dynamic projections for a moving observer are maintained using head tracking [15]. Compare also Figure 1(b).

\section{B. On the effects of $3 D$ visualizations}

Previous research has shown that $3 \mathrm{D}$ visualizations of non-realistic structures not generally lead to measurable benefits when solving visual tasks. Also, various different $3 \mathrm{D}$ cues contribute to varying degree to users' performance when solving visual tasks. The importance of motion parallax cues (due to object motion or dynamic observer position) has been demonstrated by Ware et al. [13], [15] who compared visualizations of graphs in 2D and strong 3D. In their experiments equal user performance (in terms of speed and accuracy) in 2D and 3D was still achieved for graphs with a complexity of one order of magnitude bigger in 3D. Similarly, studies on visualizations of vascular structures by van Schooten et al. [16] showed that strong $3 \mathrm{D}$ cues are important and stereoscopic cues did not have any additional enhancing effects in the presence of motion cues. These results suggest that dynamic visual cues in $3 \mathrm{D}$ visualizations might be more important than stereoscopic cues. This assumption is further supported by [17] who in a study with path tracing tasks found that stereoscopic cues only, added to a weak 3D visualizations, did not have any effect, whereas the addition of motion cues did.
In context of visualizations of maps and scalar information related to map positions few formal evaluations of $3 \mathrm{D}$ and 2D visualizations exist. In a recent study Bleisch et al. [18] compared users' discrimination of bars that were presented in a 2D map and in a 3D terrain map. No differences in time and correctness were found. Similarly, no significant effects were found in a study comparing $2 \mathrm{D}$ and $3 \mathrm{D}$ visualizations of spatio-temporal information[11]. A very recent study [19] on the estimation of distances in planar maps visualized in 2D and 3D found also no significant effects for visualization.

\section{EXPERIMENT}

From previous research we conclude that motion parallax is an important factor for exploiting three-dimensional visualizations. Motion parallax and dynamic occlusion require the visualized content to be three-dimensional geometries with sufficient spatial complexity, such as investigated in [15], [16], [17]. In visualization of maps or other tilted planar structures as studied in [12], [18], [19] the content is largely two-dimensional which might explain, why the use of 3D visualization did not gain significant effects. In the experiment presented here which directly emanates from the experiments in [18] and [19], our objective is to investigate if more complex geospatial tasks can benefit from a $3 \mathrm{D}$ representation. We also analyze if user performance is related to other factors than visualization.

\section{A. Task and stimuli}

For our experiment we chose a task that required a combinatorial assessment of two quantities - the height of bars and their distance in 2D map space. The objective was to judge relative height differences among a number of bars located in geographic positions and to evaluate them in relation to the distances between the bars. Out of a number of simultaneously visible bars participants had to decide for the pair of bars with the largest difference in height relative to their distance in map space. In a $2 \mathrm{D}$ visualization 


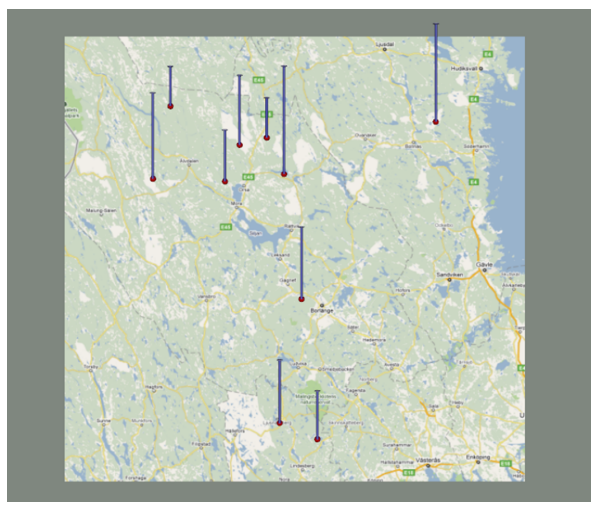

(a)

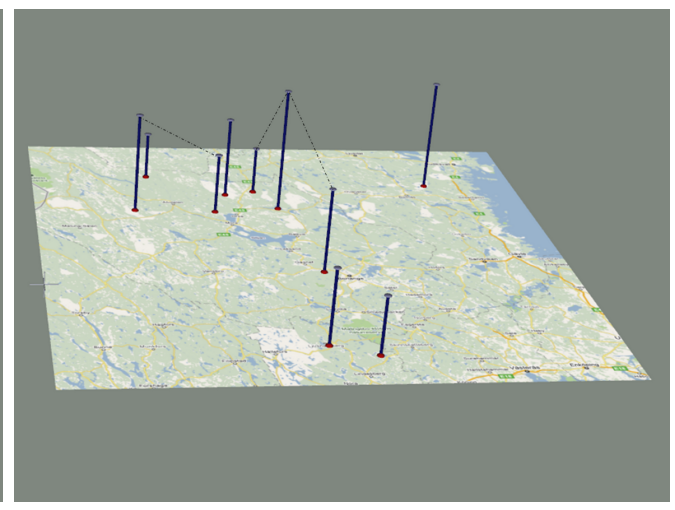

(b)

Figure 2. A stimulus containing 10 bars as used in the experiment. Stimuli were represented and visualized in $2 D$ (a) or visualized as a $3 \mathrm{D}$ slanted map with $3 \mathrm{D}$ bars in strong $3 D(\mathrm{~b})$. Imaginary lines between bar ends in sub-figure (b) are here indicated only for illustration purposes and they were not visible in the actual experiment.

this task can be considered as the evaluation of the lengths of imaginary 2D line segments and mentally judging their proportions. The same is true for a 3D presentation of bars; however, in 3D there exists an alternate approach that implies immediate assessment of the slopes of imaginary lines connecting the bar ends. In 3D visualizations there exist, quite obviously, some ambiguities as to the true slope angles of perceived lines, which in our strong 3D visualization to some extend can be resolved by interactively examining the 3D bar diagram from different viewing angles. Visual conditions tested in our experiment were $2 D$ and strong $3 D$ (according to the definition in subsection II-A). In 3D the maps were virtually slanted out off the plane of the display (55 degrees slant angle) and rendered using stereoscopic off-axis projections based on current observer positions as acquired with a camera-based tracking system. This allowed the map to be perceived correctly according to the head movement of the participants, as well as allowing for the visualization of maps with different perspectives. A visual stimulus consisted of 10 bars with varying positions within the map from which the subjects had to search and identify the pair with the largest height difference in relation to their distance in the map. Figure 2 shows one stimulus configuration visualized in $2 \mathrm{D}$ or in $3 \mathrm{D}$. Upon deciding for a candidate pair of bars, participants registered their responses by clicking with the mouse on the bottom of the two bars that they identified as the best candidates (i.e. biggest height difference in relation to distance). In order to avoid incorrect parallax in the $3 \mathrm{D}$ visualization, the $2 \mathrm{D}$ mouse cursor was replaced with a 3D cross-hair moving within the slanted map.

\section{B. Experimental design}

The experiment followed a within-subject design, that is all participants solved the task in both visualization conditions ( $2 D$ and strong $3 D$ ). To avoid carry-over effects between repeated trials, the order of visualization conditions (2D-3D or 3D-2D) was determined by random and balanced so that $50 \%$ of the test population started with either of the two conditions. Each trial comprised 25 stimuli; hence subjects viewed 25 stimuli in the $2 \mathrm{D}$ condition and viewed the same 25 stimuli in the 3D condition, and vice versa. Hereby the same stimuli configurations were used for both visualization conditions.

In order to evaluate potential learning effects within each trial (and visualization condition) stimuli for each trial were in the subsequent statistical analysis grouped into two blocks with block 1 comprising the first 13 stimuli and block 2 containing the last 12 stimuli. Since the 25 different stimuli had varying degrees of difficulty, their order of appearance within each trial was randomly assigned to counterbalance for potential differences in difficulty between both blocks. This implies that subjects did see the stimuli not in the same order.

\section{Apparatus and procedure}

The experimental apparatus consisted of a stereoscopic LCD display (Zalman ZM-M215WGD ${ }^{T M}$ ), with alternating polarization on even and odd lines. The screen size was $47.8 \mathrm{~cm}$ horizontally and $26.8 \mathrm{~cm}$ vertically with a display resolution of $1920 \times 1080$. In 3D mode the vertical resolution of each stereo-frame corresponds to half of the physical display resolution. An IR LED was attached to the center of the passive stereo glasses in order to facilitate camera based head position tracking. The tracking device (WorldViz PPT $4^{T M}$ ) was composed of 4 cameras and it was calibrated every day during the experimental phase. Thereby, the local coordinate system of the display was co-registered with the coordinate system of the tracking device.

The test group in our experiment, both teachers and students from the University of Gävle, comprised 18 subjects (12 males and six females) and their age ranged between 18 
and 65 years. The data initially collected were task response times in seconds and correctness of the response per stimulus. Accuracy was then calculated as the percentage correct answers for per subject and block of stimuli. The performed visual task was a combinational task that involved evaluation of the differences of bar heights and the assessment of their distances in the map. The task was performed in both 2D (mono) and 3D (stereoscopic) with head-tracking.

\section{Questionnaire}

In addition to the primary data (time and accuracy) other factors that might influence the visual task were gathered with a questionnaire given to the participants at the end of the test. This questionnaire intended to collect information regarding familiarity with 3D displays (yes/no), perceived visual discomfort (yes/no), strongest confidence in answers (2D/3D), and if the subject was a frequent player of 3D video-games (yes/no). Participants were also asked to make a self-assessment of their 3D vision capabilities (high/low).

\section{RESUlts}

The primary data consisted of 18 (subjects) x 25 (stimuli per trial) $\mathrm{x} 2$ (visualization conditions) time and error (correct/incorrect) responses. Individual stimuli responses were aggregated by averaging the first block of 13 observations and the second block of 12 observations for each trial. Those resulting 18 (subjects) x 2 (blocks) $\mathrm{x}$ 2 (visualizations) averaged time and accuracy observations were subsequently analyzed using the statistical package $\mathrm{R}$ [20]. Accuracy is hereby the proportion of correct responses to the total responses per subject and block.

\section{A. Times and accuracy for visualizations}

Inspection of the histograms of accuracies in 2D and 3D suggested that data were normally distributed in both cases. This was also verified with a Shapiro-Wilk test. Figure 3 shows boxplots for accuracy (a) and times (b) in the two visualization conditions 2D and 3D. Mean accuracy in 2D $(\bar{x}=0.527)$ was similar to, but slightly lower than in 3D $(\bar{x}=0.551)$. A Welch two-sample t-test with a hypothesis of equal means in the different visualization conditions showed that this difference was not significant $(\mathrm{n}=18, \mathrm{t}=-0.4951$, df $=69.937$, $\mathrm{p}$-value $=0.6221$ ).

In regard to times subjects responded faster when working with the $2 \mathrm{D}$ visualization $(\tilde{x}=9.74 \mathrm{sec}$.) than in $3 \mathrm{D}(\tilde{x}=11.21 \mathrm{sec}$.$) . Again, the hypothesis of equal$ means of times in the different visualization conditions was tested. Time observations in these kinds of psycho-physical experiments are known to be not normally distributed. Instead of applying a transformation on times on uncertain premises, we chose in all subsequent analyses of response times to employ a Wilcoxon rank sum test on the nontransformed time observations. For the comparison of $2 \mathrm{D}$ and 3D we obtained no significant differences $(\mathrm{n}=18, \mathrm{~W}$ $=501, \mathrm{p}$-value $=0.0992$ ).

\section{B. Learning effects}

Regarding potential effects of learning between successive trials, we performed a two-way ANOVA on the accuracy observations with visualization and presentation order as factors. This analysis did not reveal any significant effect for either factor nor any interaction effect. As the ANOVA is not applicable for the observed times, we compared times from the groups that started $2 \mathrm{D}$ or $3 \mathrm{D}$, respectively, using a Wilcoxon rank sum test. Also this comparison revealed no difference between trials.

Significant learning effects could in part be observed within both visualization conditions in a block-wise comparison of the observations for block pre (stimuli 1-13) and block post (stimuli 14-25). Although the boxplots for accuracies in Figure 3(c) suggest better accuracy in the second block of stimuli in both visualizations, those differences were not significant for the $2 \mathrm{D}$ condition nor in the 3D condition. However, in regard to response times clear improvements in the post blocks can be seen in both visualization conditions (see Figure 3(d)). The apparent reductions of times between blocks were significant both in 2D $\left(\mathrm{n}_{1}=13, \mathrm{n}_{2}=12, \mathrm{~W}=238, \mathrm{p}\right.$-value $\left.=0.01557\right)$ and in $3 \mathrm{D}$ $\left(\mathrm{n}_{1}=13, \mathrm{n}_{2}=12, \mathrm{~W}=281, \mathrm{p}\right.$-value $\left.<0.001\right)$.

\section{Personal abilities and skills}

According to test answers from the questionnaires, we compared responses for different subject groups. From 18 subjects $\mathrm{n}_{1}=12$ rated that their spatial vision capabilities as low, whereas $\mathrm{n}_{2}=6$ rated them as high. Figure 4 shows boxplots for accuracy (a) and times (b) in both groups. The group stating high confidence in their 3D vision capabilities achieved clearly better accuracy both in the 2D visualization and in the 3D visualization (Figure 4(a)). This difference in accuracy was significant in 2D $(\mathrm{t}=2.172, \mathrm{df}=31.049$, $\mathrm{p}$ value $=0.03761)$, as well as in $3 \mathrm{D}(\mathrm{t}=2.4045, \mathrm{df}=33.27$, $\mathrm{p}$ value $=0.02192$ ). The same holds true for times, where the confident group showed shorter response times (Figure 4(b)). This difference in time was, however, only significant in the $2 \mathrm{D}$ visualization condition $\left(\mathrm{n}_{1}=12, \mathrm{n}_{2}=6, \mathrm{~W}=77\right.$, pvalue $=0.0241$ ).

Visual discomfort, that is eye strain and possibly associated dizziness or headache, is another factor frequently reported in studies investigating 3D visualizations. In our experiment as many as $\mathrm{n}_{1}=8$ subjects, or $44 \%$, reported visual discomfort. $\mathrm{N}_{2}=10$ subjects did not. A comparison of achieved accuracy and times in the 3D visualization condition is provided in Figure 5(a) and (b). Although there were no statistically significant differences between both groups it is notable that the group that experienced visual discomfort 


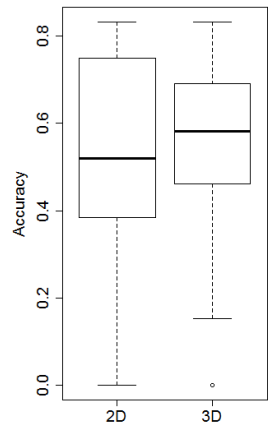

(a)

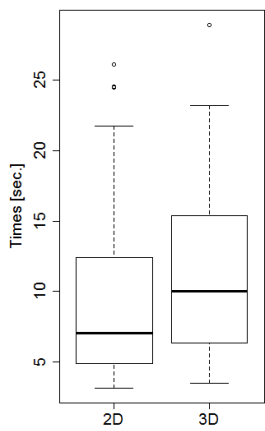

(b)

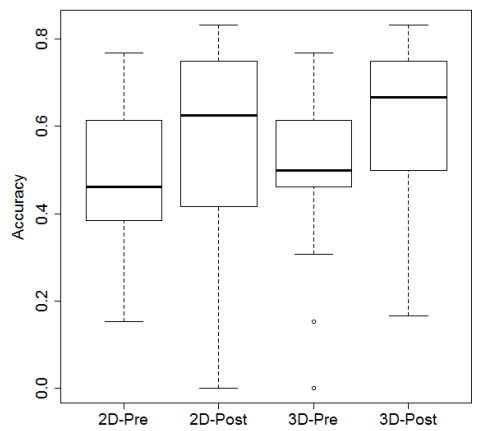

(c)

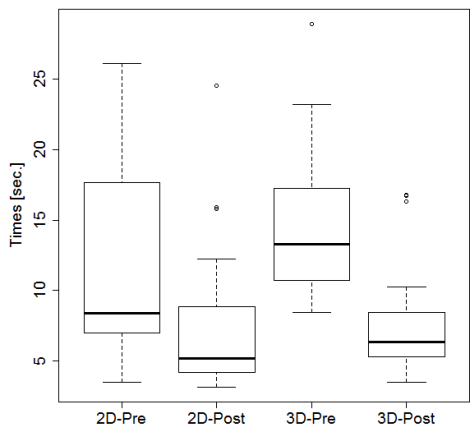

(d)

Figure 3. Box plots for accuracy (a) and times (b) for 2D and 3D. Sub-figures (c) and (d) show boxplots of the accuracies and times for blocks (pre and post) in $2 \mathrm{D}$ and $3 \mathrm{D}$.

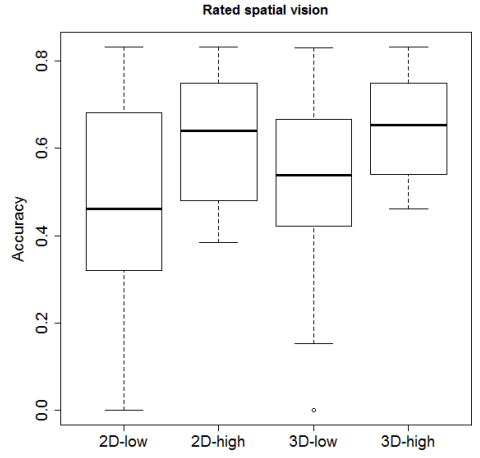

(a)

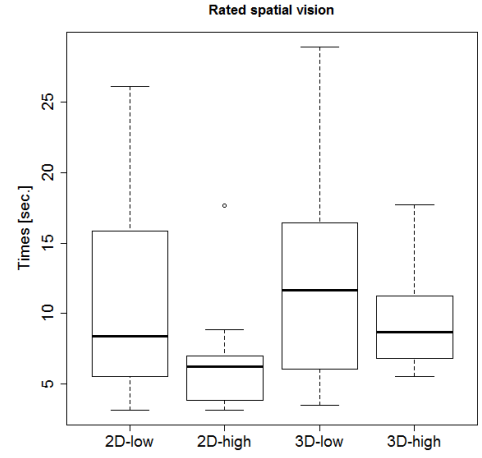

(b)

Figure 4. Results for groups rating their 3D/spatial capability as low and high. Accuracies (a) and times (b) are presented separately for both visualization conditions.

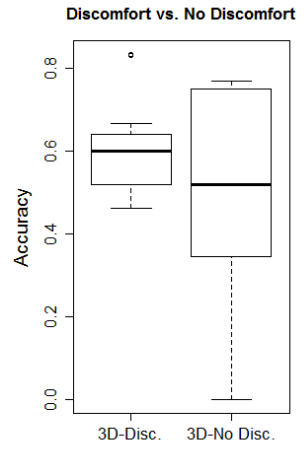

(a)

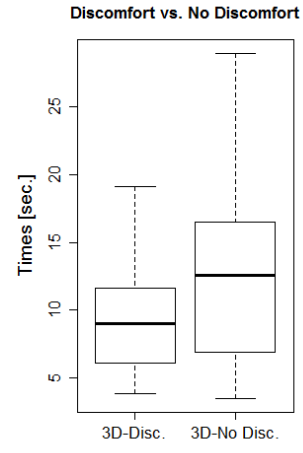

(b)

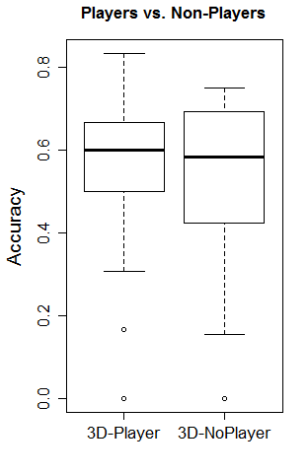

(c)

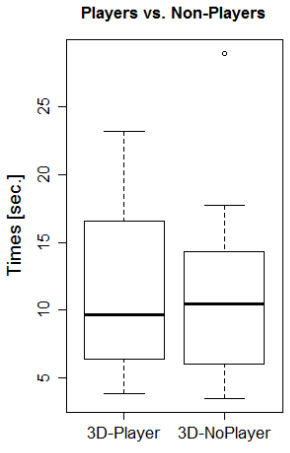

(d)

Figure 5. Results in the 3D visualization for groups experiencing visual or not in 3D (a) and (b). Sub-figures (c) and (d) show results for the groups of frequent game players and non-players. 
performed better. The means of accuracy for the group with visual discomfort $\left(\bar{x}_{\text {Discom fort }}=0.602\right)$ and without visual discomfort $\left(\bar{x}_{N o-D i s c o m f o r t}=0.510\right)$ differ by $18 \%$. The median of times differs even by $38 \%\left(\tilde{x}_{\text {Discom fort }}=9.02\right.$ and $\tilde{x}_{N o-D i s c o m f o r t}=12.54$ ).

A final finding concerns frequent game playing which was reported by $\mathrm{n}_{1}=6$ subjects, or $33 \%$. The boxplots in Figure 5 show, that somehow unexpected, there were no differences in accuracy (c) or times (d) between both groups in the $3 \mathrm{D}$ visualization condition.

\section{CONCLUSION AND DISCUSSION}

Our experimental study investigated potential benefits of a 3D map representation with 3D bars for the relative assessment of quantitative data in context of geographic distance. The main results of this experiment indicate neither improved accuracy nor shorter task solution times for the $3 \mathrm{D}$ visualization condition. Although previous investigations of similar, albeit simpler, geospatial tasks prefigure such results [18], [19], we expected yet to observe some clear differences in this study. Contrary to the work presented in [18], [19] we employed a more complex spatial assessment task that required estimation of two different metrics and their relative ratios. In a $2 \mathrm{D}$ visualization of bars, this task must be performed as a two-stage sequence requiring short term memory. The 3D visualization of bars provided an alternative, immediate, cue to directly assess these ratios; the slope of imaginary lines connecting bar ends in relation to ground. In our study we did no follow-up if subjects actually followed this strategy. Therefore it cannot be ruled out that participants in our study used the same strategy to solve the task as in 2D. Yet on the other hand, in our questionnaire we asked test participants which of the two visualizations they felt most confident with in regard to their performance. Only three $(17 \%)$ voted for $2 \mathrm{D}$, whereas the remaining 15 subjects $(83 \%)$ felt more confident with the 3D visualization. Considering, again, that almost half of the population felt visual discomfort in $3 \mathrm{D}$, these results confirm what appears to be a prevalent overestimation of the real benefits of threedimensional visualizations.

Still it must be assumed that the assessment of the slope of an imaginary 3D line over a ground plane is ambiguous in a projective display. In providing strong 3D cues (mainly stereoscopy and motion-parallax) in our 3D visualization we hoped to alleviate this problem, but we have no evidence as to how much participants in our study exploited these cues.

Self-assessments of individual 3D capabilities were reliable. Subjects who rated their spatial skills high performed indeed better on the tasks, not only in the 3D part but also in $2 \mathrm{D}$. Contrary to other research that speaks about increased 3D and spatial skills of people who are frequent game players, we found the opposite. In our study frequent players did apparently not achieve any different performance in the 3D task compared with non-players.

On a final note we conclude that the results of this study are in line with those of a number of previous works in the field of geospatial visualization which do not point out strong advantages of $3 \mathrm{D}$ visualizations for the type of spatial tasks investigated. On the other hand there is ample evidence in the literature that threedimensional visualizations are effective for other types of tasks (interpretation of tree-like graphs) and that dynamic spatial cues in $3 \mathrm{D}$ visualization play a significant role. Our continued research in geospatial visualization will therefore be geared towards further exploring other, more efficient visual mappings of geospatial data that better exploit those cues.

\section{ACKNOWLEDGEMENT}

The authors would like to thank the participants for their willingness to participate in this study.

\section{REFERENCES}

[1] Johnson, A.; Leigh, J.; Morin, P. \& Van Keken, P. (2006), 'GeoWall: Stereoscopic Visualization for Geoscience Research and Education', Computer Graphics and Applications, IEEE 26(6), 10-16.

[2] Slocum, T. A.; Blok, C.; Jiang, B.; Koussoulakou, A.; Montello, D. R.; Fuhrmann, S. \& Hedley, N. R. (2001), 'Cognitive and Usability Issues in Geovisualization', Cartography and Geographic Information Science 28:1, 61-75.

[3] Kirschenbauer, S.Dykes, J.; MacEachren, A. \& Kraak, M.J., ed., (2005), Exploring Geovisualization, Elsevier, chapter Applying "True 3D" Techniques to Geovisualization: An Empirical Study, pp. 363-387.

[4] Kröninger, A. \& Bartel, S. (1998), '3d-Gis for Urban Purposes', Geoinformatica 2(1), 79-103.

[5] Paar, P. (2005), 'Landscape visualizations: Applications and requirements of $3 \mathrm{D}$ visualization software for environmental planning', Comput., Environ. and Urban Systems 30, 815839.

[6] Shepherd, I. D. H.Dodge, M. \& Turner, M., ed., (2007), GeoVisualization, Wiley, chapter Travails in the third dimension: a critical evaluation of 3D geographical evaluation, pp. 199222 .

[7] Tufte, E. R. (1997), Visual Explanations - Images and Quantities, Evidence and Narrative, Graphics Press.

[8] Hägerstrand, T., 'What about people in regional science?', Regional Science Association Papers 24, 7-21.

[9] Andrienko, N. V.; Andrienko, G. L. \& Gatalsky, P. (2003), 'Exploratory spatio-temporal visualization: an analytical review', Journal of Visual Languages and Computing 14, 503541. 
[10] Pettersson, L. W.; Mats, M.; Spak, U. \& Seipel, S. (2005), Visualizations of Symbols in a Horizontal Multiple Viewer 3D Display Environment, in 'Proceedings of the Ninth International Conference on Information Visualisation', IEEE Computer Society, Washington, DC, USA, pp. 357-362.

[11] Kjellin, A.; Pettersson, L. W.; Seipel, S. \& Lind, M. (2008), 'Evaluating 2D and 3D visualizations of spatiotemporal information', ACM Trans. Appl. Percept. 7(3), 19:1-19:23.

[12] Lind, M.; Seipel, S. \& Mattiason, C. (2001), 'Displaying meta-information in context', Behaviour and Information Technology 20(6), 427-432.

[13] Ware, C. \& Mitchell, P. (2005), Reevaluating stereo and motion cues for visualizing graphs in three dimensions, in 'Proceedings of the 2nd symposium on Applied perception in graphics and visualization', ACM, New York, NY, USA, pp. 51-58.

[14] Kjellin, A.; Pettersson, L. W.; Seipel, S. \& MatsLind (2009), 'Different levels of 3D: An evaluation of visualized discrete spatiotemporal data in space-time cubes', Inf Visualization 9(2), 152-164.

[15] Ware, C. \& Mitchell, P. (2008), 'Visualizing graphs in three dimensions', ACM Trans. Appl. Percept. 5(1), 2:1-2:15.

[16] van Schooten, B. W.; van Dijk, E. M. A. G.; ZudilovaSeinstra, E.; Suinesiaputra, A. \& Reiber, J. H. C. (2010), The effect of stereoscopy and motion cues on 3D interpretation task performance, in 'Proceedings of the International Conference on Advanced Visual Interfaces', ACM, New York, NY, USA, pp. 167-170.

[17] van Beurden, M.; Kuijsters, A. \& IJsselsteijn, W. (2010), 'Performance of a path tracing task using stereoscopic and motion based depth cues' 'Second International Workshop on Quality of Multimedia Experience (QoMEX)', 176-181.

[18] Bleisch, S.; Dykes, J. \& Nebiker, S. (2008), 'Evaluating the Effectiveness of Representing Numeric Information Through Abstract Graphics in 3D Desktop Virtual Environments', Cartographic Journal 45(3), 216-226.

[19] Seipel, S. (2012), 'Evaluating 2D and 3D Geovisualizations for Basic Spatial Assessment', Behaviour and Information Technology,(in press)

[20] Ihaka R. \& Gentleman R. (1996), 'R: A Language for Data Analysis and Graphics', Journal of Computational and Graphical Statistics 5(3), 299-314. 